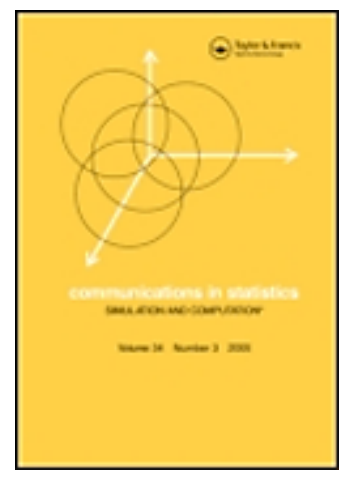

\title{
Output dead-time in point processes
}

\begin{tabular}{|c|c|}
\hline Journal: & Communications in Statistics - Simulation and Computation \\
\hline Manuscript ID: & LSSP-2008-0289.R2 \\
\hline Manuscript Type: & Original Paper \\
\hline $\begin{array}{r}\text { Date Submitted by the } \\
\text { Author: }\end{array}$ & 29-Jul-2009 \\
\hline Complete List of Authors: & $\begin{array}{l}\text { PICINBONO, Bernard; Laboratoire des signaux et systèmes, } \\
\text { Supélec }\end{array}$ \\
\hline Keywords: & $\begin{array}{l}\text { Point processes, Statistical modelling, Dead-time, Renewal } \\
\text { processes, Poisson processes }\end{array}$ \\
\hline Abstract: & $\begin{array}{l}\text { Dead-time effects modify the statistical properties of point } \\
\text { processes. For their analysis we define a point process by the } \\
\text { intervals between successive points and we calculate their } \\
\text { transformation by dead-time. It cannot be expressed in closed form } \\
\text { but we show that it can be written in a recursive form. Using this } \\
\text { recursion, various statistical properties of point processes with } \\
\text { dead-time are analyzed. We focus on the probability distribution of } \\
\text { the intervals between points and the coincidence function } \\
\text { describing the second-order properties. For the rare processes } \\
\text { where calculations are possible there is an excellent agreement } \\
\text { between computer experiments and theory. }\end{array}$ \\
\hline \multicolumn{2}{|c|}{$\begin{array}{l}\text { Note: The following files were submitted by the author for peer review, but cannot be converted } \\
\text { to PDF. You must view these files (e.g. movies) online. }\end{array}$} \\
\hline PICINBONO.zip & \\
\hline
\end{tabular}




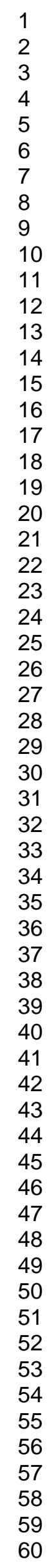

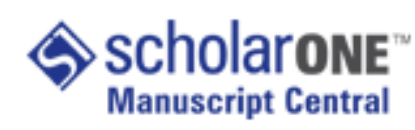
7 


\title{
Output dead-time in point processes
}

\author{
B. Picinbono \\ Laboratoire des Signaux et Systèmes ${ }^{1}$, \\ Supélec, Plateau de Moulon, 91190 Gif-sur-Yvette, France \\ Tel: (33)-(1)-69.85.17.40, Email : bernard.picinbono@lss.supelec.fr \\ July 2009
}

\begin{abstract}
When events of (time) temporal point processes are too close to each other they can be erased by dead-time effects. Among various possible mechanisms of dead-time, the output dead-time is the most important. Dead-time effects modify the statistical properties of point processes and some of these modifications are (analyzed) analyzed in this paper. To do so, we note that a point process is defined by the distance between its successive points called life-time which (constitute) constitutes a discrete time positive signal. The dead-time mechanism is a system which transforms such a signal into another (of the same nature) discrete time positive signal. Except in very specific cases this transformation cannot be expressed in closed form. We show however that it can be written in a recursive form analogous to the state representation of systems. By using this recursion, various statistical properties of point processes with dead-time are analyzed in computer experiments. In this study we focus on the probability distribution of the intervals between points and the coincidence function which describes the second-order properties of the point process. For the rare processes where theoretical calculations are possible there is an excellent agreement between experiment and theory.
\end{abstract}

Some key words. Point processes, statistical modeling, signal representation, nonlinear signal processing, life-time.

\footnotetext{
${ }^{1}$ Unité mixte de recherche (UMR 8506) du Centre national de la recherche scientifique (CNRS), de l'École supérieure d'électricité (Suplec) et de l'Université de Paris-Sud 11 (UPS).
} 


\section{Introduction}

Dead-time (DT) effects appear in a large number of physical phenomena and, in the time domain, they are the counterpart of band limitation in the frequency domain. As any physical system cannot pass arbitrarily high frequencies, so it cannot distinguish too close time instants.

The purpose of this paper is to analyze DT effects on Point Processes (PP). Such effects have been discussed in many papers originating from different scientific fields such as, for example, Particle Physics [Delotto et al. (1964), Muller (1973), Muller (1974)], Optical Communications [Bédard (1967), Saleh (1977), Teich and Vanucci (1976), Vanucci and Teich (1981)] or Signal Processing [Gruner and Johnson (2001)].

(In its principle the) The problem can be stated as follows: the DT effect transforms an input PP $\mathcal{P}$ into an output PP $\mathcal{P}^{\prime}$ according to a specific mechanism in which some points of $\mathcal{P}$ are erased. The problem is then to deduce some properties of $\mathcal{P}^{\prime}$ from the structure of the transformation and from the statistics of $\mathcal{P}$. Except in some very specific cases, the complete theoretical solution of this problem is almost impossible to obtain. As a consequence we are obliged to limit the theoretical analysis to certain elementary properties and to carry out an experimental analysis (for example) by computer simulation.

A (time) temporal PP is a sequence of random instants and the distances between successive points are positive random variables (RV) called life-times. These distances can be considered as the values of a discrete time positive random signal $x_{i}$ and the statistical properties of the PP are equivalent to those of $x_{i}$. In particular if the signal $x_{i}$ is strictly white, which means that the RVs $x_{i}$ are independent and identically distributed (IID), the PP is a renewal $\mathrm{PP}$.

(After the DT effect the input PP $\mathcal{P}$ defined by $x_{i}$ is transformed into an output PP $\mathcal{P}^{\prime}$ defined by another signal $y_{i}$, and the transformation of $x_{i}$ into $y_{i}$ is defined by the DT mechanism.)

The input PP $\mathcal{P}$ defined by $x_{i}$ is transformed by the DT effect which erases some of its points into another $\mathrm{PP} \mathcal{P}^{\prime}$ called output PP. This PP is defined by another signal $y_{i}$, and the transformation of $x_{i}$ into $y_{i}$ is defined by the DT mechanism. Our first purpose is to establish the input-output relationship of the system which yields $y_{i}$ from $x_{i}$. This transformation is in 
general (extremely) non-linear which makes it impossible to calculate all the properties of $y_{i}$. This is the reason why the only possible approach is the use of computer experiments to analyze the problem.

The paper is organized as follows. In Section 2 we set the problem out in detail and introduce some appropriate notations for its solution. In Section 3 we establish the relation between input and output for a specific model of DT called output DT which is the most important for practical applications. These relations are presented in the form of recursive equations and are similar to the state equations used in (system) systems theory [see p. 193 of Picinbono (1988)]. These recursions are non-linear and, except in some very specific cases, the statistical properties of the output $y_{i}$ cannot be calculated from those of the input $x_{i}$. When theoretical calculations are impossible computer experiments using these recursions are (in general more easily realized) usually feasible. In particular, using this procedure, we can obtain some (expectation) expected values of the output $y_{i}$ and also its marginal probability density function (PDF). In Sections 4 and 5 we present computer experiments with output DT in order to visualize the transformation due to the DT. If theoretical calculations are possible there is an excellent agreement with experimental results. Note that the approach presented in this paper is similar to the one appearing in [Igarashi (1994)], but the calculations are completely different.

Finally it is worth pointing out the relation between DT and the (queues) queuing problems appearing in service systems. However the approach is quite different because the purpose of service systems is to avoid loss of messages while it is not possible to avoid losses of points in (presence) the presence of DT [Bedekar and Azizoglu (1998), Gelenbe and Pujolle (1987), Hajek (1984), Krishnan (1990)].

\section{Statement of the problem and (notations) notation}

Consider a stationary $\mathrm{PP}$ in time $\mathcal{P}$ with (density) intensity $\mu$. Its points $T_{i}$ are time instants $t_{i}$. In order to simplify the presentation, we use in what follows the expression "point $t_{i}$ " to express "point $T_{i}$ at time $t_{i}$ ". A PP can be considered as a sequence of positive RVs which are the values of the intervals between successive points. These intervals are called life-times of order 1 . Let 
$x_{i}$ be the RVs describing $\mathcal{P}$ defined by $x_{i}=t_{i}-t_{i-1}$, which implies that $x_{i}>0$.

The set of RVs $x_{i}$ can also be considered as a positive discrete-time stationary random signal and the statistical properties of the PP $\mathcal{P}$ are entirely defined from those of this signal. However the calculation of properties such as the probability $p_{n}(T)$ of counting $n$ points $T_{i}$ in an interval $[t, t+T[$ is in general rather complicated [Cox and Isham (1980), Saleh (1977)] and in most cases only a numerical approach is possible.

The DT effect erases some of these points and this yields a new PP of points $\theta_{j}$ noted $\mathcal{P}^{\prime}$. The (density) intensity $\lambda$ of $\mathcal{P}^{\prime}$ (satisfies obviously) obviously satisfies $\lambda \leq \mu$. In order to associate to each $t_{i}$ a well defined $\theta_{i}$ we proceed as follows. If $t_{i}$ is not erased we have $\theta_{i}=t_{i}$. Suppose furthermore that $t_{i+1}$ is erased. In this case we state $\theta_{i+1}=\theta_{i}=t_{i}$. This means that it is possible for a point $\theta_{i}$ to come from distinct points $t_{i}$ and it is said that $\theta_{i}$ is a multiple point. (In such case we use the expression of multiple point $\theta_{i}$.) Let $y_{i}=\theta_{i}-\theta_{i-1}$ be the distance between successive points $\theta_{i}$. (It) This is a non-negative RV which takes the value 0 if $\theta_{i}$ is a multiple point. In conclusion the DT mechanism associates to each $t_{i}$ at least one $\theta_{i}$, and thus to each positive $x_{i}$ one well defined non-negative $y_{i}$.

There are several mechanisms of DT effects which transform $x_{i}$ into $y_{i}$. The two most important are the input and the output DTs.

In the input DT each point $t_{i}$ of $\mathcal{P}$, erased or non-erased, generates a DT interval $\left[t_{i}, t_{i}+D[\right.$ and all the points of $\mathcal{P}$ arriving in this interval are erased. It is clear that when the (density) intensity $\mu$ increases, $\lambda$ decreases, and it even tends to zero if $\mu \rightarrow \infty$, which corresponds to the classical congestion phenomenon where all the points of $\mathcal{P}$ are erased and $\mathcal{P}^{\prime}$ disappears. This input DT effect is sometimes called (type II counter) the type II counter in the statistical literature [see p. 101 of Cox and Isham (1980)]. It is analyzed in some detail in Picinbono (2007), and henceforth no longer discussed.

The output DT, sometimes called (type I counter) the type I counter, is more important for the applications and does not present the congestion phenomenon introduced just above. It is characterized by the fact that each point $\theta_{i}$ of $\mathcal{P}^{\prime}$ generates a DT interval $\left[\theta_{i}, \theta_{i}+D[\right.$ where $D$ is a positive constant characterizing the length of the DT, (and) such that all the points $t_{i}$ of $\mathcal{P}$ in this interval are erased. This means that only the non-erased points of $\mathcal{P}$ 
contribute to the DT effect. In this case if $\mu \rightarrow \infty$ the PP $\mathcal{P}^{\prime}$ tends to become a periodic process, or a sequence of points [with a constant interval $D$ between them) $\theta_{i}$ such that the corresponding distances $y_{i}$ are equal to 0 or to $D$.

To each of these two DT effects one can associate a particular mechanism for the transformation of the RVs $x_{i}$ into the RVs $y_{i}$. The explicit calculation of this transformation and of its consequences for the properties of the $y_{i} \mathrm{~s}$ is in general extremely complicated and in most cases only experimental approaches are possible.

Let us present the kind of problems that can be analyzed. The $\operatorname{PP} \mathcal{P}$ is completely defined by the statistical properties of the $x_{i}$ s. The simplest case appears when these RVs are IID. This means that $x_{i}$ is a discrete-time strictly white noise. In this situation the $\mathrm{PP} \mathcal{P}$ is a renewal $\mathrm{PP}$ and all of its statistical properties can be deduced from the Probability Density Function (PDF) of the $x_{i}$ s. If this $\mathrm{PDF}$ is exponential, $\mathcal{P}$ is a Poisson process, which is the simplest model of PP. But the assumption that the $x_{i}$ s are IID is often too restrictive and we shall present examples of situations in which it is not (introduced) required.

\section{Dead-time input-output relationships}

Our purpose is to study the transformation of $\mathcal{P}$ into $\mathcal{P}^{\prime}$ (after) induced by DT effect. The problem is (then) to calculate the system which generates the signal $y_{i}$ from the signal $x_{i}$ for a given (mechanism of the DT effect) DT mechanism. We shall write this system in a non-linear recursive form, analogous to a state representation of non-linear systems, and present some consequences of this recursive description.

Consider a point $t_{i}$ of $\mathcal{P}$ which is not erased. Let $t_{i+p}$ be the first point of $\mathcal{P}$ (posterior to) following $t_{i}$ which is not erased. This means that $p$ is the (first) smallest positive integer such that either $x_{i+p}>D$ or $x_{i+p}<D$ but $\sigma_{i, p}=x_{i+1}+x_{i+2} \ldots+x_{i+p}>D$. It is this last relation which ensures that, even if all the points of $\mathcal{P}$ satisfy $x_{i}<D$, some of them are not erased because the sum $\sigma_{i, p}$ is greater than $D$. This is the case when $\mu \rightarrow \infty$ which yields that $\mathcal{P}^{\prime}$ becomes a periodic process of period $D$. The algorithm generating the $y_{i} \mathrm{~s}$ from the $x_{i} \mathrm{~s}$ is decomposed into two parts similar to the state equation and the observation equation, allowing to deduce the output of a system from its 
input.

For this purpose we introduce a signal $s_{i}$, called the state signal in analogy with the state vector used in system theory. It is defined as follows: it increases and returns to zero when $t_{i}$ is not erased, which appears either when $x_{i}>D$ or when $s_{i}>D$. It is then defined by the recursion

$$
s_{1}=u\left(D-x_{1}\right) x_{1} \quad, \quad s_{i+1}=u\left(D-x_{i+1}\right) u\left(D-s_{i}-x_{i+1}\right)\left(s_{i}+x_{i+1}\right),
$$

where $u($.$) is the unit step function. As a result of the definition of the output$ DT the signal $y_{i}$ is given by the recursion

$y_{1}=u\left(x_{1}-D\right) x_{1} \quad, \quad y_{i+1}=\left[u\left(x_{i+1}-D\right)+u\left(D-x_{i+1}\right) u\left(s_{i}+x_{i+1}-D\right)\right]\left(s_{i}+x_{i+1}\right)$.

Indeed if $x_{i+1}>D$, the point $t_{i+1}$ is not erased and $y_{i+1}=s_{i}+x_{i+1}$. On the other hand if $x_{i+1}<D$, the point $t_{i+1}$ is erased (uniquely) if $s_{i}+x_{i+1}<D$.

In order to visualize the behaviour of the complete algorithm, we present the results for nine values of $x_{i}, s_{i}$ and $y_{i}$ obtained in a computer experiment in which $x_{i}$ is uniformly distributed in $[0,1]$ for the value $D=0.75$ of the DT.

$\begin{array}{cccccccccc} & 1 & 2 & 3 & 4 & 5 & 6 & 7 & 8 & 9 \\ x_{i} & 0.4120 & 0.7446 & 0.2679 & 0.4399 & 0.9334 & 0.6833 & 0.2126 & 0.8392 & 0.6288 \\ s_{i} & 0.4120 & 0 & 0.2679 & 0.7079 & 0 & 0.6833 & 0 & 0 & 0.6288 \\ y_{i} & 0 & 1.1566 & 0 & 0 & 1.6412 & 0 & 0.8959 & 0.8392 & 0\end{array}$

The erased points $t_{i}$ are $t_{1}, t_{3} t_{4}, t_{6}$, and $t_{9}$. The points not erased by the fact that $x_{i}>D$ are $t_{5}$ and $t_{8}$ and those not erased by the effect of the sum are $t_{2}$ and $t_{7}$. Note that $s_{i}$ satisfies $s_{i}<D$. Indeed suppose that $s_{i}<D, x_{i}<D$, and $s_{i+1}=s_{i}+x_{i+1}>D$. It results from (1) that $s_{i+1}=0$.

Let $m_{x}$ and $m_{y}$ be the mean values of $x_{i}$ and $y_{i}$ respectively. These(s) quantities are related to the densities $\mu$ and $\lambda$ of the PPs $\mathcal{P}$ and $\mathcal{P}^{\prime}$ respectively by

$$
\mu=1 / m_{x} \quad ; \quad \lambda=1 / m_{y} .
$$

The mean value $m_{x}$ is given in the definition of the PP $\mathcal{P}$. The mean value $m_{y}$ can easily be deduced from the algorithms (1) and (2). Indeed it results directly from these algorithms that

$$
\sum_{i=1}^{N} x_{i}=\sum_{i=1}^{N} y_{i} \triangleq S_{N}
$$


where $N$ is the number of variables $x_{i}$ analyzed in the experiment, or also the number of steps of the recursions (1) and (2). The experimental estimation of $m_{x}$ and $m_{y}$ are then

$$
\hat{m}_{x}=S_{N} / N \quad ; \quad \hat{m}_{y}=S_{N} / M_{N}
$$

where $M_{N}$ is the number of variables $y_{i}$ that are positive in the experiment. It then suffices to calculate the number of $y_{i}$ s taking the value 0 , say $N_{0}$, and $M_{N}=N-N_{0}$. These values appear clearly in Table 1 , and their number $N_{0}$ is deduced very simply from the sequence of the $y_{i}$.

From the sequences $x_{i}$ or $y_{i}$ it is possible to deduce one of the most important features concerning the PPs $\mathcal{P}$ and $\mathcal{P}^{\prime}$ which is their marginal PDF. In particular, as noted above, in the case of renewal PPs all their statistical properties can be deduced from this PDF. There are various ways for the measurement of the PDF of a sequence of RVs and in what follows we use a procedure starting from the histograms of the sequences $x_{i}$ or $y_{i}$. (As we shall see this yields excellent results in the case in which the analytical expression of the PDF is known.) In the cases in which the analytical expression of the PDF is known we shall verify an excellent agreement between analytical and experimental results, which constitutes a test of the validity of the procedure.

For various calculations it is also interesting to know the marginal PDF of the so called lifetime (or) of higher order. The life time of order $n$ of a PP is the distance between a point of the process and the $n$th point of the process ( posterior to) following it. (If $n=1$ we return to the lifetime already introduced.) For renewal PPs these lifetimes do not yield new statistical information because the lifetimes of order $n$ are sums of IID RVs and their (PDF) PDFs (is a multiple convolution) are multiple convolutions of the (PDF) PDFs of the intervals between successive points (defining completely) completely specifying the PP. These convolutions are however often difficult to be obtained analytically, and then their experimental measurement yields (an) interesting information on the PP.

The PDF $f_{n}(x)$ of the lifetime of order $n$ of a PP defined by the stationary signal $x_{i}$ appears in the expression of the coincidence function $c(x)$ which (describes) completely specifies the second order properties of the PP, and in particular its spectrum as defined in Bartlett (1962) or in Cox and Isham 
(1980). The coincidence function of a PP is defined by the relation

$$
\operatorname{Pr}\left\{[d N(\theta)=1] \cap\left[d N\left(\theta^{\prime}\right)=1\right]\right\}=c\left(\theta-\theta^{\prime}\right) d \theta d \theta^{\prime},
$$

where $d N(\theta)$ is the number of points of the $\mathrm{PP}$ appearing in the interval $[\theta, \theta+d \theta[$. Some of its properties and the principle of its measurement are discussed in Picinbono (2008) and here we limit the presentation to the most fundamental properties. The basic result concerning the coincidence function $c(x)$ is expressed by

$$
c(x)=\lambda \sum_{n=1}^{\infty} f_{n}(|x|),
$$

where $\lambda$ is the (density) intensity of the PP and $f_{n}(x)$ the PDF of the lifetime of order $n$ of the PP. This expression is used for the measurement of the coincidence function as indicated in Picinbono (2008). The use of multiple histograms allows us to measure the PDFs $f_{n}(x)$ for a finite number $M$ of lifetimes and we estimate $c(x)$ by using a finite sum of $M$ terms instead of a series. The precision and the performance of the method is analyzed in Picinbono (2008). In the cases in which $c(x)$ can be calculated analytically the results of measurements are in excellent agreement with those of the calculations. Note finally that for regular ergodic processes the coincidence function tends to $\lambda^{2}$ when $x$ tends to infinity. This results from (6) and from the fact that for such processes the RVs $d N(\theta)$ and $d N\left(\theta^{\prime}\right)$ become independent when $\theta-\theta^{\prime}$ tends to infinity and satisfy $\operatorname{Pr}[d N(\theta)=1]=\mathrm{E}[d N(\theta)]=\lambda d \theta$, which is one of the definitions of the (density) intensity of a PP.

In the case of PPs obtained from output DT there is an additional property which greatly simplifies the measurements of the coincidence function. Indeed the fundamental property of the distances $y_{i}$ between points after output DT is that they satisfy $y_{i}>D$. This implies that the PDF $f_{1}(y)$ is equal to 0 if $y<D$. Similarly the PDF $f_{k}(y)$ is equal to 0 if $y<k D$. This implies that the series (7) is always a finite sum of terms. There is one term only if $D<y<2 D$, and $k$ terms only if $k D<y<(k+1) D$.

\section{Output dead-time and Poisson processes}

As indicated previously, Poisson processes are the simplest and also the most important examples of PPs. Furthermore the complete calculation of the statistics (after output DT) of the PP obtained after DT effect is rather simple 
and it is (almost the only) a rare example where theory and experiment can easily be compared.

A Poisson PP of (density) intensity $\mu$ is defined by the fact that the RVs $x_{i}$ are IID with an exponential distribution

$$
f(x)=\mu u(x) \exp (-\mu x)
$$

(After output DT we obtain) The PP obtained after DT effect is a renewal process and, as shown in the Appendix, the PDF of the lifetime $y_{i}$ is

$$
f_{1}(y)=u(y-D) \mu \exp [-\mu(y-D)]
$$

where $D$ is the (value) duration of the DT. This implies that $f_{1}(y)=f(y-D)$, which means that it is simply a shifted exponential distribution. For $D=0$, $y_{i}=x_{i}$ and we return to (8). We deduce from (8) and (9) that

$$
m_{x}=1 / \mu \quad ; \quad m_{y}=(1+\mu D) / \mu=m_{x}+D
$$

The PDF of the lifetime of order $n$ after DT is obtained from multiple convolution of (9) and the result is

$$
f_{n}(y)=u(y-n D) \mu \frac{[\mu(y-n D)]^{n-1}}{(n-1) !} \exp [-\mu(y-n D)] .
$$

We shall now (verify) compare these results to those obtained by by computer simulations. The Poisson process $\mathcal{P}$ is simulated by generating a sequence of $N$ IID exponential RVs. For (simplification) simplicity we assume in most of our experiments that the (density) intensity $\mu$ of $\mathcal{P}$ is 1 . The number $N$ is usually of the order of $10^{6}$ or $10^{7}$, which yields (a) good statistical precision.

The (estimation) estimator $\hat{m}_{y}$ (of $m_{y}$ ) compared to its theoretical value $m_{y}=1+D$ for various values of $D$ is presented in the following table.

$\begin{array}{ccccccc}D & 0 & 0.5 & 1 & 1.5 & 2 & 3 \\ m_{y} & 1 & 1.5 & 2 & 2.5 & 3 & 4 \\ \hat{m}_{y} & 1.0001 & 1.4995 & 2.0001 & 2.5002 & 2.9997 & 3.9996\end{array}$

This shows an excellent agreement between theory and experiment.

Let us now present some results on the PDFs and on the coincidence (functions) function (measurements) estimation. In Figure 1 we present results concerning the PDFs of the ( lifetime) lifetimes of order 1 to 4 of the PP obtained 
from a Poisson process of (density) intensity 1 after output DT of value $D=1$. The points are the results of the (measurements) estimation of these PDFs deduced from normalized histograms. The continuous curves represent the PDFs defined by (11). We observe that the experimental points correspond exactly to the theoretical values. This agreement can be considered as a test of the correct behaviour of the algorithm yielding the lifetimes $y_{i}$ after dead time.

In Figure 2 we present experimental results concerning $f_{1}(y)$ for various values of $D$. The (measurements) estimations of $f_{1}(y)$ are presented in semilogarithmic coordinates, in such a way that the experimental points must be located on parallel straight lines corresponding to (9). This clearly appears in the figure.

In Figure 3 we present (measurements) estimations of the coincidence function for two values of $D$. As previously the points correspond to experimental (measurements) estimations and the continuous curves are deduced from (7) and (11). Here also we observe an excellent agreement between theory and experiment which confirms the validity of the algorithms calculating the output dead time and the principle of the (measurements) estimations of the coincidence function. We note also that the asymptotic value of $c(x)$ is effectively $\lambda^{2}=1 / m_{y}^{2}$ equal to 0.444 and 0.25 for $D=0.5$ and $D=1$ respectively.

\section{Output dead-time and some renewal processes}

As indicated previously, renewal processes are PPs in which the distances between successive points are IID random variables. This means that the statistical properties of such processes are completely defined by the PDF $f(x)$ of the lifetime of order one. Note incidentally that Poisson processes are also renewal processes in which $f(x)$ is given by (8). We shall now consider the case of certain particular renewal processes which are particularly interesting for some applications.

\subsection{Erlang processees}

Erlang PPs are renewal processes for which the PDF of the life time is

$$
p(x)=4 \mu^{2} x \exp (-2 \mu x) .
$$


The quantity $\mu$ is the (density) intensity of the PP, inverse of the mean value of the life time. It is another rare example where the PDF of the life time after DT can be explicitly calculated and, as shown in the Appendix, the result is

$$
f_{1}(y)=\mu u(y-D) \exp [-2 \mu(y-D)] g(y)
$$

where $g(y)=A y+B$ with

$$
A=2 \mu[1+\exp (-4 \mu D)] ; B=1-2 \mu D-(1+2 \mu D) \exp (-4 \mu D)
$$

Note that (13) and (14) show clearly that, unlike in the case of Poisson processes, the DT effect is not simply reduced to a shift of the PDF (12).

The results of computer experiments appear in Figure 4 for various values of $D$ and $\mu=1$. Note that high values of $D$ are analyzed because they (appear effectively) are practically used in some neural computers [McNeill and Card (2005)]. The continuous curves are calculated from the previous equations and the points correspond to the experimental (measurements) estimations (in an experiment) with $10^{6}$ samples of the RXs $x_{i}$. As for Poisson processes, there is an excellent agreement between theory and experiment. We note in this figure that for $D>2$ the PDFs are approximately obtained by the shifting of the PDF $f_{1}(y)$ obtained for $D=2$. This is due to the fact that the term $\exp (-4 \mu D)$ in $(14)$ is almost zero.

The mean value of the lifetime after output dead-time defined by the PDF (13) and (14) is given by

$$
m_{y}=3 /(4 \mu)+D+[1 /(4 \mu)] \exp (-4 \mu D)
$$

For large values of $D$ this mean value is approximately equal to $3 /(4 \mu)+D$. In the following table we present some values of $m_{y}$ given by (15) and experimental results $\hat{m}_{y}$ for the same values of $D$ for an Erlang PP of (density) intensity $\mu=1$. We observe, as in the case of Poisson processes, an excellent agreement between theory and experimental results.

$\begin{array}{cccccccc}D & 0 & 0.5 & 1 & 2 & 3 & 4 & 5 \\ m_{y} & 1 & 1.2838 & 1.7546 & 2.7501 & 3.7500 & 4.7500 & 5.7500 \\ \hat{m}_{y} & 1.0001 & 1.2839 & 1.7545 & 2.7495 & 3.7491 & 4.7490 & 5.7488\end{array}$

Results concerning the (measurements) estimations of coincidence functions are presented in Fig. 5. For $D=0$, i.e. for an Erlang PP of (density) 
intensity $\mu$ without DT, the coincidence function is $c(x)=\mu^{2}[1-\exp (-4 \mu|x|)]$. It is (represented in the) represented by a continuous line in the figure and it fits with the experimental points quite well. For nonzero DT there is no simple expression (of) for the coincidence function. The constant straight lines calculated for $D=0.5$ and $D=1$ are the functions $c(x)=1 / m_{y}^{2}$ presented for $1.5<x<4$, where $m_{y}$ is given by (15). As indicated above, we verify that the coincidence function tends to $\lambda^{2}$, where $\lambda=1 / m_{y}$ is the (density) intensity of the PP after DT.

\subsection{Renewal Processes with Uniform Distribution}

In this section we suppose that the RVs $x_{i}$ are IID and uniformly distributed in $[0,2[$. This value is chosen in order to make a comparison with the other PPs analyzed above. All these PPs have the same mean value $\left(m_{x}=1\right)$, which implies the same (density) intensity $\lambda=1$.

The measured PDFs $f_{1}(y)$ for various values of the DT appear in Fig. 6 . The analytic expressions of these PDFs are very complicated but the experimental results highlight the following points.

At first it is clear that, as in the case of Erlang processes and contrary to the case of Poisson processes, the PDF of the life time after output DT is not obtained by a simple shift of the PDF without DT.

It appears also that the PDFs $f_{1}(y)$ of $y_{i}$ after DT are zero outside the intervals $\left[D, D+2\left[\right.\right.$. This can be explained quite simply. Indeed let $t_{i}$ be a non-erased point of $\mathcal{P}$. The first non-erased point (posterior to) following $t_{i}$ cannot be outside the interval $\left[t_{i}+D, t_{i}+D+2[\right.$. This comes from the fact that the distance between successive points $t_{i}$ of the initial PP is smaller than 2 , because of its uniform distribution in $[0,2[$.

For $D<2$ the PDF $f_{1}(y)$ is constant in the interval $[D, 2]$ and decreases approximately linearly in the interval $[2, D+2]$. This means that there is (in general) with high probability only one point erased and as a result the (lifetimes) lifetime $y_{i}$ is a sum of two IID RVs $x_{i}$, which introduces a triangular PDF. Finally we observe that for $D=4$, the $\operatorname{PDF} f_{1}(y)$ is approximately equal to $(1 / 2)(-y+6)$ in the interval $[4,6]$ and zero otherwise. This structure appears still more clearly for $D>4$ and the PDF tends to be equal to $(1 / 2)(-y+D+2)$ in the interval $[D, D+2]$ and zero otherwise. This leads to a mean value equal 
to $D+2 / 3$.

This can be verified by the experimental (measurement) estimation of the mean value yielding the results presented in the following table.

$\begin{array}{ccccccccc}D & 0 & 0.5 & 1 & 2 & 3 & 4 & 5 & 10 \\ \hat{m}_{y}-1 & 0.0001 & 0.2840 & 0.6486 & 1.7183 & 2.6572 & 3.6714 & 4.6654 & 9.6665\end{array}$

We observe that $\hat{m}_{y}$ does not vary linearly in terms of $D$ for $D<3$, as it was the case with a Poisson PP. On the contrary we have approximately $\hat{m}_{y}=D+0.66$ when $D>3$.

In Fig. 7 we present some experimental results concerning the coincidence function for two values of $D$ : 0.5 and 1 , as in figures 2 and 4 . There is no closed form expression of this function and we can only highlight some particular points. As indicated above the coincidence function $c(x)$ is zero for $x<D$ and equal to $\lambda f_{1}(x)$ when $D<x<2 D$. We verify these points by comparing them with the PDFs presented in Fig. 5. As in Fig. 4 we have shown the straight line $f(x)=\lambda^{2}$ for $2<x<4$, where $\lambda$ is the (density) intensity of the PP after DT equal to the inverse of the mean value $m_{y}$ presented in the previous table. We observe in Fig. 6 that, in accordance with the theory, $c(x)$ tends to $\lambda^{2}$ for large values of $x$.

\section{Output dead-time and EAR(1) processes}

The assumption of independence between successive life times, which is the basis of the theory of renewal PPs, is too restrictive in many applications and various models of PPs with correlated time intervals have been presented. Among them, the best known is the exponential autoregressive (EAR) of order one PP first introduced in [Jacobs and Lewis, 1977] and also discussed in [Cox and Isham, 1980].

We shall (fist) first briefly recall its structure before discussing its properties when output DT appears. Consider the signal $X_{k}$ defined by the recursion

$$
X_{k}=a X_{k-1}+B_{k} Z_{k}
$$

in which $B_{k}$ is a Bernoulli white noise, or a sequence of IID RVs taking only the values 0 and 1 with the probabilities $1-\alpha$ and $\alpha$ respectively and $Z_{k}$ is a positive strictly white noise, or a sequence of IID positive RVs, independent of the $B_{k} \mathrm{~s}$ and with an exponential marginal distribution. This equation is 
similar to the recursion defining autoregressive models, and the parameter $a$ is the regression coefficient. One can show that if $\alpha=1-a$ the RVs $X_{k}$ defined by this recursion are positive and with an exponential marginal distribution. They can then be used as the lifetimes of PPs. The marginal distribution of these lifetimes are then the same as for Poisson processes, but the Poisson character disappears because these lifetimes are no longer independent and their covariance function $\gamma_{n}$ is $\sigma_{X}^{2} a^{|n|}$, where $\sigma_{X}^{2}$ is the variance of $X_{k}$. The interest of this model of PP is its analogy with the Poisson process, in particular if $a=0$, it becomes a Poisson process.

The statistical properties of this model of PP are very difficult to obtain in simple mathematical expressions, and this is especially true when DT effects appear. On the other hand the computer simulation of this model is especially simple and we shall now present some experimental results obtained with the method used above in the case of renewal PPs.

In the following table we present the results of (measurements) estimations of the mean value of the life time for various values of the DT $D$ and of the regression coefficient $a$. The experimental mean value is obtained from approximately $10^{6}$ samples of points of an EAR(1) process of (density) intensity $\mu=1$. The first line obtained for $a=0$ corresponds to a Poisson PP for which the mean value after output DT must be equal to $D+1$. The results are similar to those presented above in the section devoted to Poisson processes.

$\begin{array}{cccccccc}a \backslash D & 0 & 0.5 & 1 & 2 & 3 & 4 & 5 \\ 0 & 1.0002 & 1.5005 & 2.0006 & 3.0001 & 4.0012 & 4.9992 & 6.0001 \\ 0.5 & 1.0015 & 1.4332 & 1.9128 & 2.9275 & 3.9552 & 4.9709 & 5.9822 \\ 0.7 & 0.9994 & 1.4062 & 1.8715 & 2.8882 & 3.9266 & 4.9525 & 5.9684 \\ 0.9 & 0.9995 & 1.3726 & 1.8187 & 2.8213 & 3.8775 & 4.9275 & 5.9558\end{array}$

We observe (on the columns) in each of the columns of this table that the influence of the regression coefficient $a$ is rather small, especially for large values of $D$. For a better understanding of this point we shall now present results on (PDFs measurements) PDF estimations.

Some results are presented in Fig. 8. The points correspond to (measurements) estimations of the PDFs $f_{i}(y), 1 \leq i \leq 4$ of the lifetimes of order $i$ after an output DT of value $D=1$ and a regression coefficient $a=0.7$. The continuous curves are those appearing in Fig. 1 concerning the effect of the same DT on a Poisson process of the same (density) intensity. We observe 
that for $i=1$ the effect of the DT is rather small(, which explains the results of mean values (measurements) estimations appearing in the previous table). Note that when there is no DT $(D=0)$ the PDF $f_{1}(y)$ is exponential and does not depend on $a$. On the other hand, the effect of the correlation between successive intervals on the PDF of their sum is considerable and this appears clearly in the difference between the continuous curves and the experimental points. (Such an effect has an influence on the coincidence function because of (7) and (measurements) estimations of this function are presented in Fig. 9 for $a=0.5$ ). This appears also on the structure of the coincidence function because of (7) and results on its estimation appear in Fig. 9 for $a=0.5$. They must be compared to those of Fig. 3 obtained with the same values of the parameters but for $a=0$, which corresponds to a Poisson process. This comparison highlights clearly the influence of the correlation between successive intervals on the form of the coincidence function.

\section{Appendix: calculations for Poisson and Erlang processes}

Let $\mathcal{P}$ be a Poisson process of (density) intensity $\mu$. Consider an arbitrary non-erased point of this process chosen as the origin of the time axis. It results from the definition of the output DT that all the points of $\mathcal{P}$ appearing in the interval $[0, D[$ are erased because there is a non-erased point at the origin. As a consequence the first point of $\mathcal{P}^{\prime}$ posterior to the origin is located in the interval $[y, y+d y[$ iff there is no point of $\mathcal{P}$ in the interval $[D, y[$ and at least one point of $\mathcal{P}$ in $[y, y+d y[$. This yields (9). The generating function associated with this $\mathrm{PDF}$ is $G_{1}(s)=\exp (-s D) \mu /(\mu+s)$. It is clear that $\mathcal{P}^{\prime}$ is a renewal $\mathrm{PP}$. The generating function corresponding to the life time of order $n$ is then $G_{n}(s)=\left[G_{1}(s)\right]^{n}$. By taking its inverse Laplace transform we obtain (11).

Consider now the case of Erlang processes. There are many ways to define them and the simplest one is by using the method of periodic erasing on stationary Poisson processes. Consider such a PP with (density) intensity $2 \mu$ and let denote $t_{i}$ its points. The PP with points $t_{2 i}$, which means the PP deduced from the Poisson process by erasing all the points $t_{2 i+1}$, is an Erlang process. Its (density) intensity is clearly $\mu$ and it results immediately from this definition that the PDF of the intervals between successive points is given by (12) which is the PDF of a sum of two IID exponential random variables. 
Consider now the effect of the output DT on such a PP. As for the calculation in the case of a Poisson process, suppose that there is a non-erased point at the origin. Let $\mathcal{A}, \mathcal{B}$ and $\mathcal{C}$ be the intervals $[0, D[,[D, y[$, and $[y, y+d y[$ respectively, which implies $y>D$. Because of the construction of the Erlang PP $\mathcal{P}$ from a Poisson process of (density) intensity $2 \mu$ noted $\mathcal{P} \mathcal{O}$, the first point of $\mathcal{P}^{\prime}$ after the origin is in $\mathcal{C}$ either if there is an even (or zero) number of points of $\mathcal{P O}$ in $\mathcal{A}, 1$ point of $\mathcal{P O}$ in $\mathcal{B}$ and at least 1 point of $\mathcal{P O}$ in $\mathcal{C}$ or if there is an odd number of points of $\mathcal{P O}$ in $\mathcal{A}, 0$ point of $\mathcal{P O}$ in $\mathcal{B}$ and at least 1 point of $\mathcal{P O}$ in $\mathcal{C}$. By noting that the probabilities of even or odd numbers for a Poisson process are given by $(1 / 2)[1+\exp (-4 \mu D)]$ and $(1 / 2)[1-\exp (-4 \mu D)]$ respectively, we obtain (13) and (14).

\section{BIBLIOGRAPHY}

Arce G., Barner K., and Ma L. (2003). Random early detection gateway congestion control using median queue size estimates. IEEE Trans. Signal Processing, 51, 2149-2164.

Bartlett M. S. (1962), "The spectral analysis of point processes," J. Roy. Statist. Soc. B , 25, 264-296.

Bédard G. (1967). Dead-time corrections to the statistical distributions of photoelectrons. Proc. Phys. Soc., 90, 131-161.

Bedekar A. and Azizoglu M. (1998). The information-theoretic capacity of discrete time queues. IEEE Trans. Inf. Theory, 44, 446-466.

Cox D. and Isham V. (1980). Point processes. London: Chapman and Hall.

Delotto I., Manfredi P., and Principi F. (1964). Counting statistics and deadtime losses. Energ. Nuclear (Milan), 11, 557-564.

Gelenbe E. and Pujolle G. (1987). Introduction to Queueing Networks. NewYork: Wiley.

Gruner C. and Johnson D. (2001). Calculation of the Kullback-Leibler distance between $p$ models. ICASSP 2001, 3437-3440.

Hajek B. (1984). Optimal control of two interacting service stations. IEEE Trans. Aut. Control, 29, 491-499.

R. Igarashi et al.(1994). Evaluation of fluctuating dead times by time interval distribution measurement. IEEE Trans. on Instrum. and Measur., 43, 164169. 
Jacobs P. and Lewis P. (1977), "A mixed autoregressive-moving average exponential sequence and point process (EARMA 1,1) ," Adv. Appl. Prob., 9, pp. $87-104$.

Krishnan K.(1990). Joining the right queue: a state-dependent decision rule. IEEE Trans. Aut. Control, 35, 104-108.

McNeill D. and Card H. (2005). Refractory pulse counting processes in stochastic neural computers. IEEE Trans. on Neural Networks, 16, 505-508.

Muller J. (1973). Dead-time problems. Nucl. Instrum. Methods, 112, 47-67.

Muller J. (1974). Some formulae for a dead-time distorded Poisson process. Nucl. Instrum. Methods, 117, 401-404.

Picinbono B. (1988). Principles of Signals and Systems. London: Artech House.

Picinbono B. (2007). Temps mort d'entrée dans les processus ponctuels. Traitement du signal, 24, 411-428.

Picinbono B. (2008). Measurements of second-order properties of point processes. IEEE Trans. Instrumentation and Measurement, 57, 548-555.

Saleh B. (1977). Photoelectron Statistics. Berlin: Springer Verlag.

Teich M. and Vanucci G (1978). Observation of dead-time modified photocounting distributions for modulated laser radiation . J. Opt. Soc. Amer., 68, $1338-1342$.

Takagi H. (1993). Queuing Analysis: a (fundation) foundation of performance evaluation, vol 3, Amsterdam: North-Holland.

Vanucci G. and Teich M. (1981). Dead-time-modified photocount mean an variance for chaotic radiation. J. Opt. Soc. Amer., 71, 164-170. 


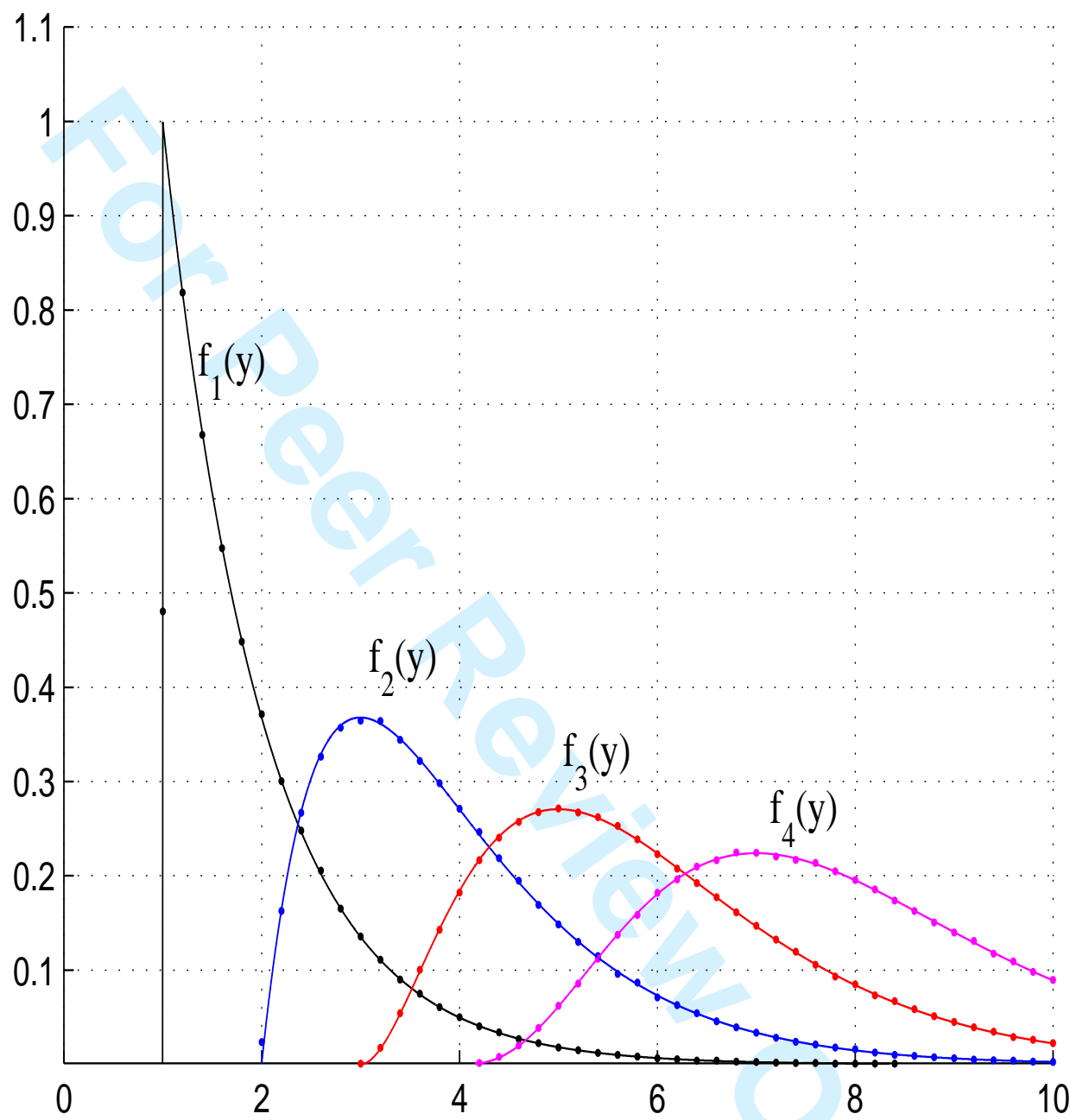

Figure 1: $\operatorname{PDFs} f_{1}(y), f_{2}(y), f_{3}(y), f_{4}(y)$ of a Poisson PP with output DT, $D=1$. 


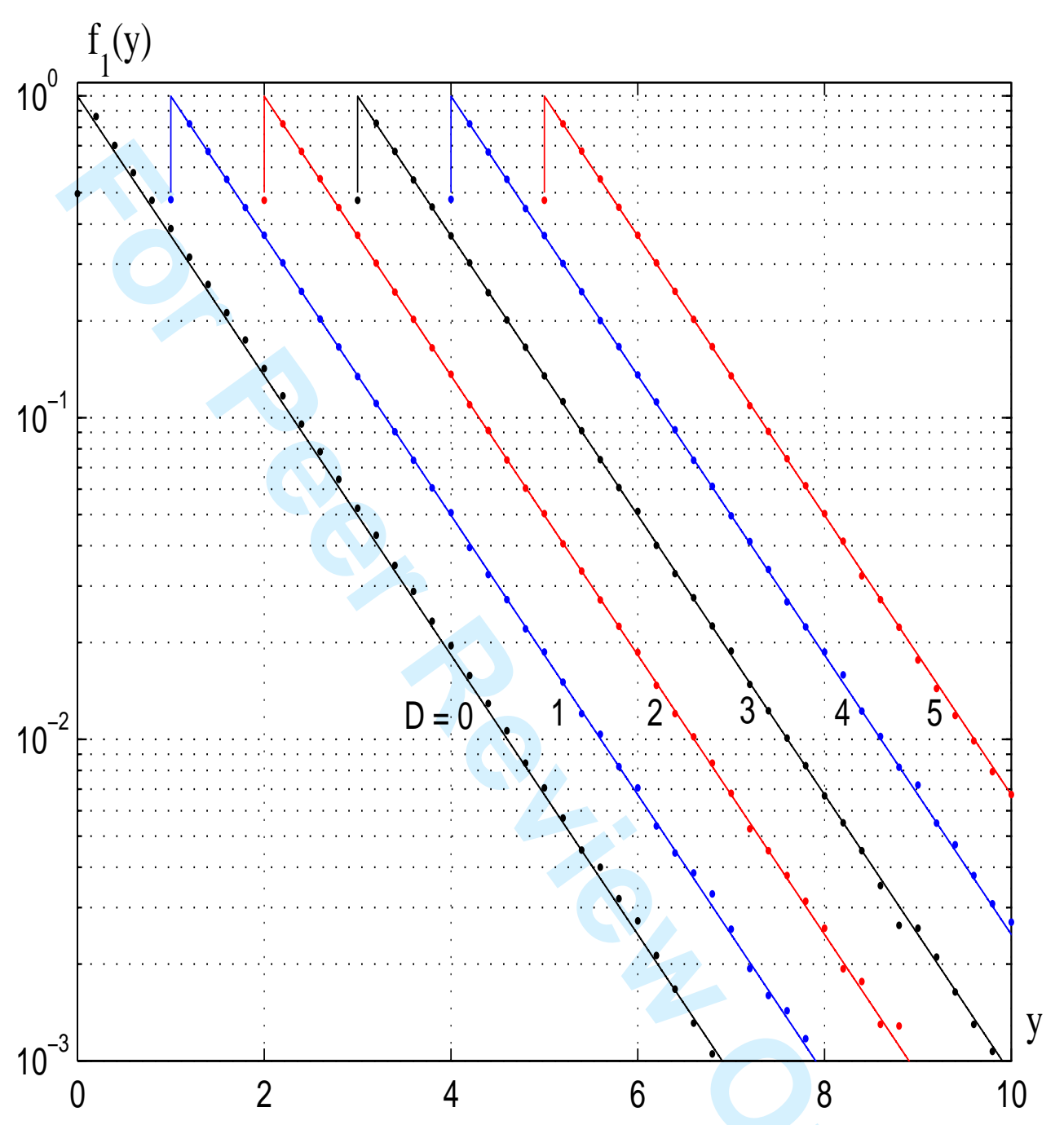

Figure 2: PDFs $f_{1}(y)$ of a Poisson PP with output DT for various values of $D$. 


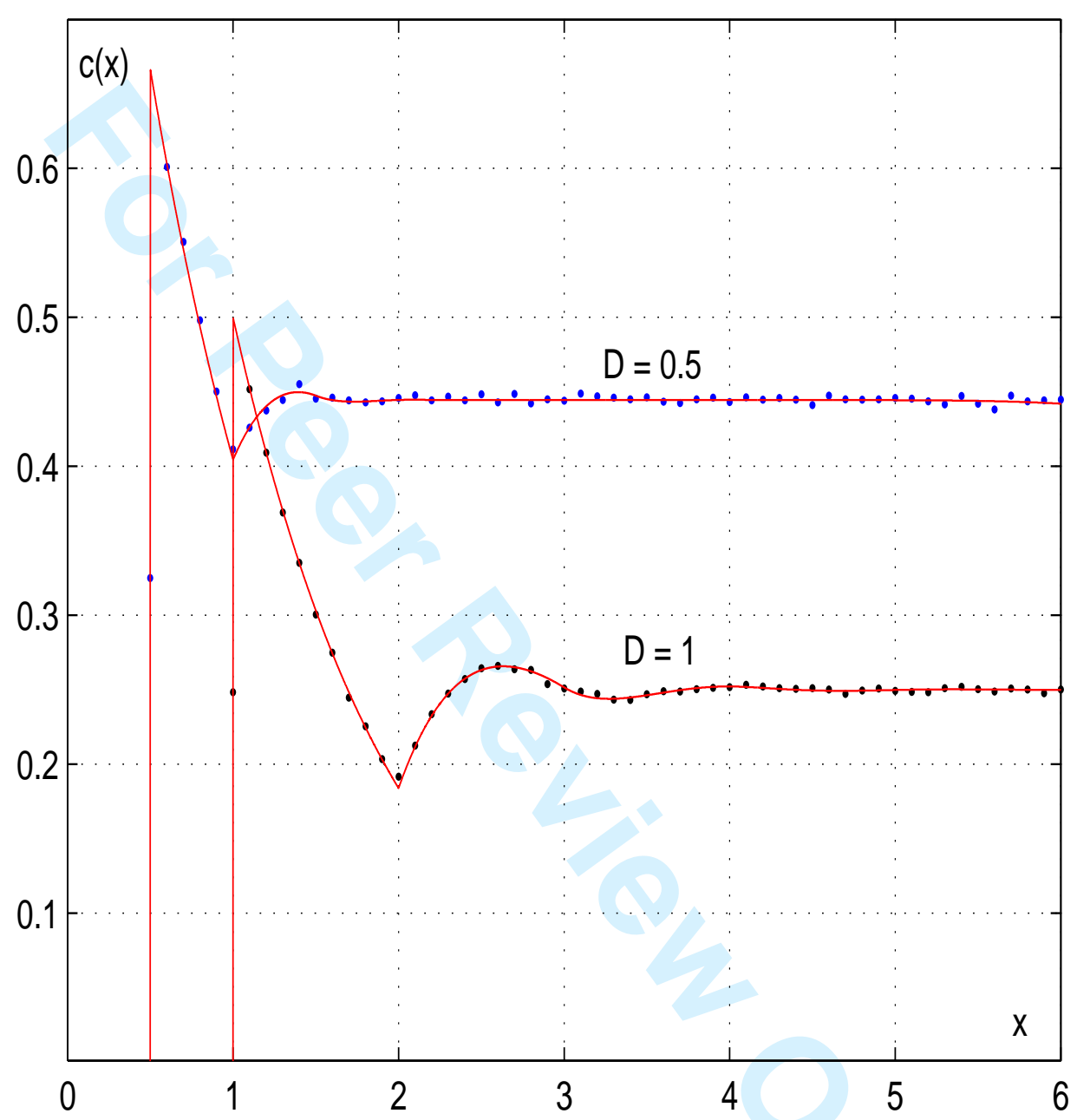

Figure 3: Coincidence function of a Poisson PP with output DT for two values of $D$. 


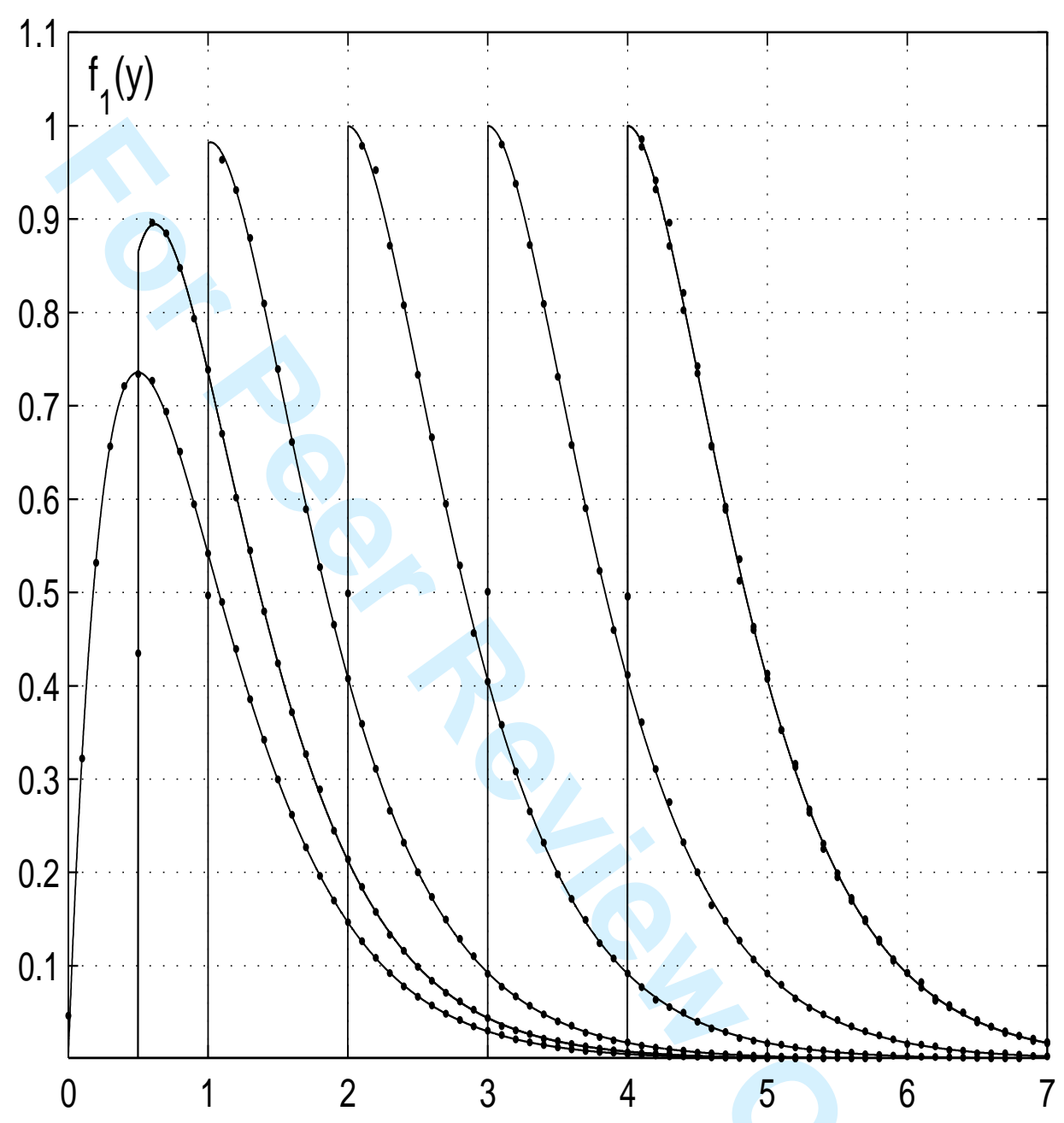

Figure 4: $\operatorname{PDF} f_{1}(y)$ of lifetime with output DT on an Erlang process for various values of $D: 0,0.5,1,2,3,4$. Points: experiments, curves: theoretical values given by (13) and (14) 


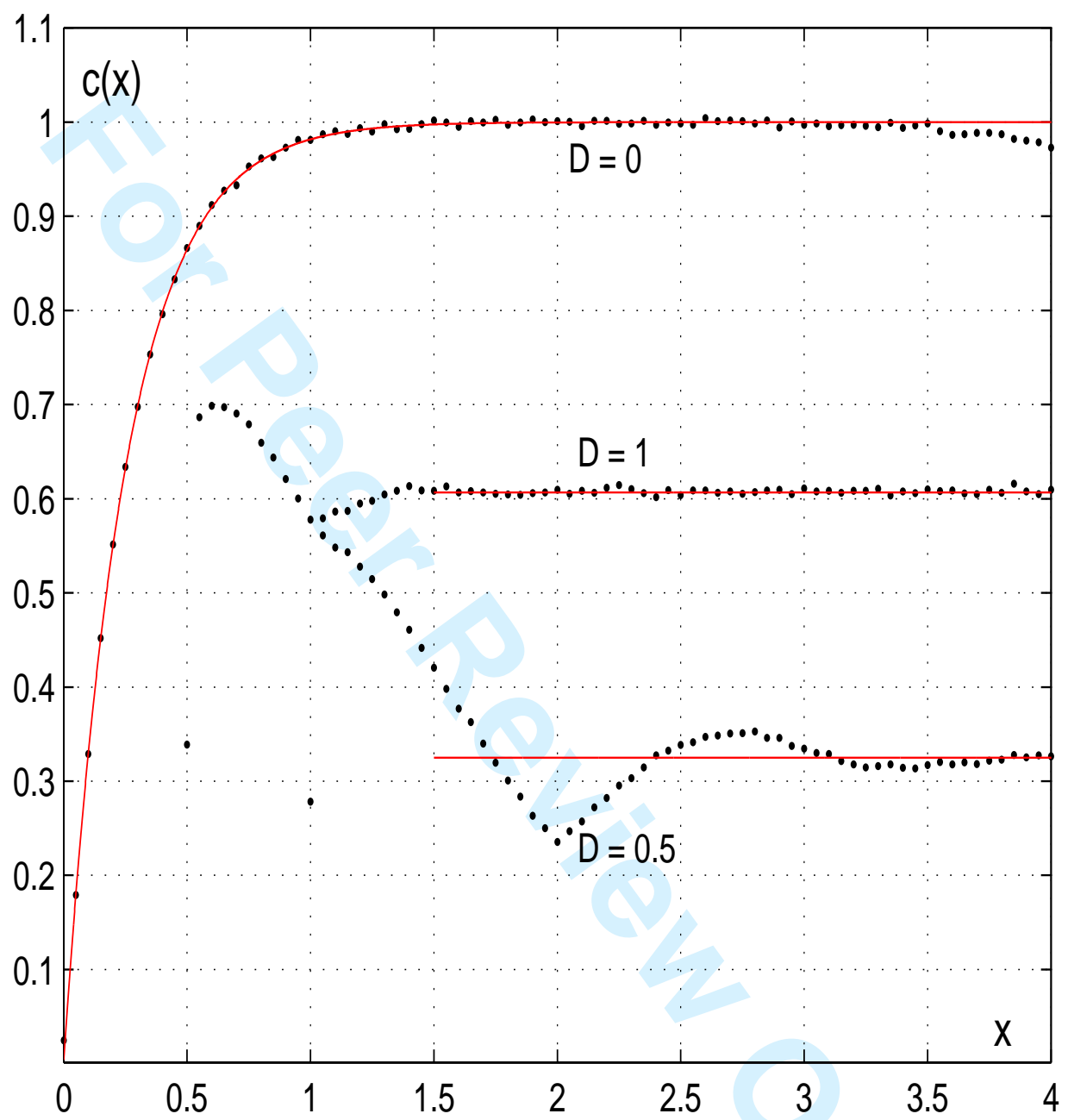

Figure 5: Coincidence function of an Erlang PP with output DT for two values of $D$. 

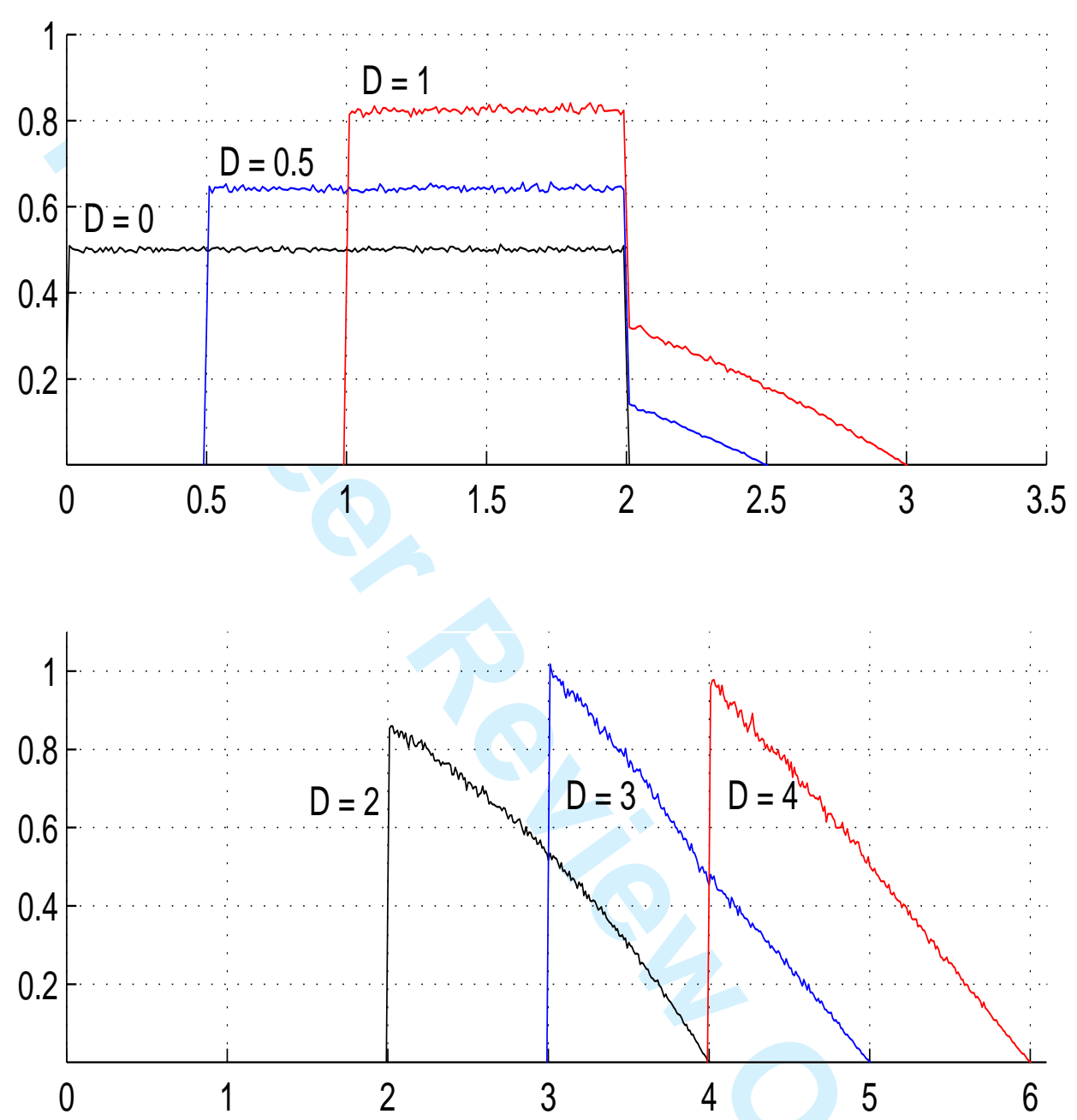

Figure 6: $\operatorname{PDF} f_{1}(y)$ of lifetime with output DT on a PP with uniform lifetime for various values of $D: 0,0.5,1,2,3,4$. 


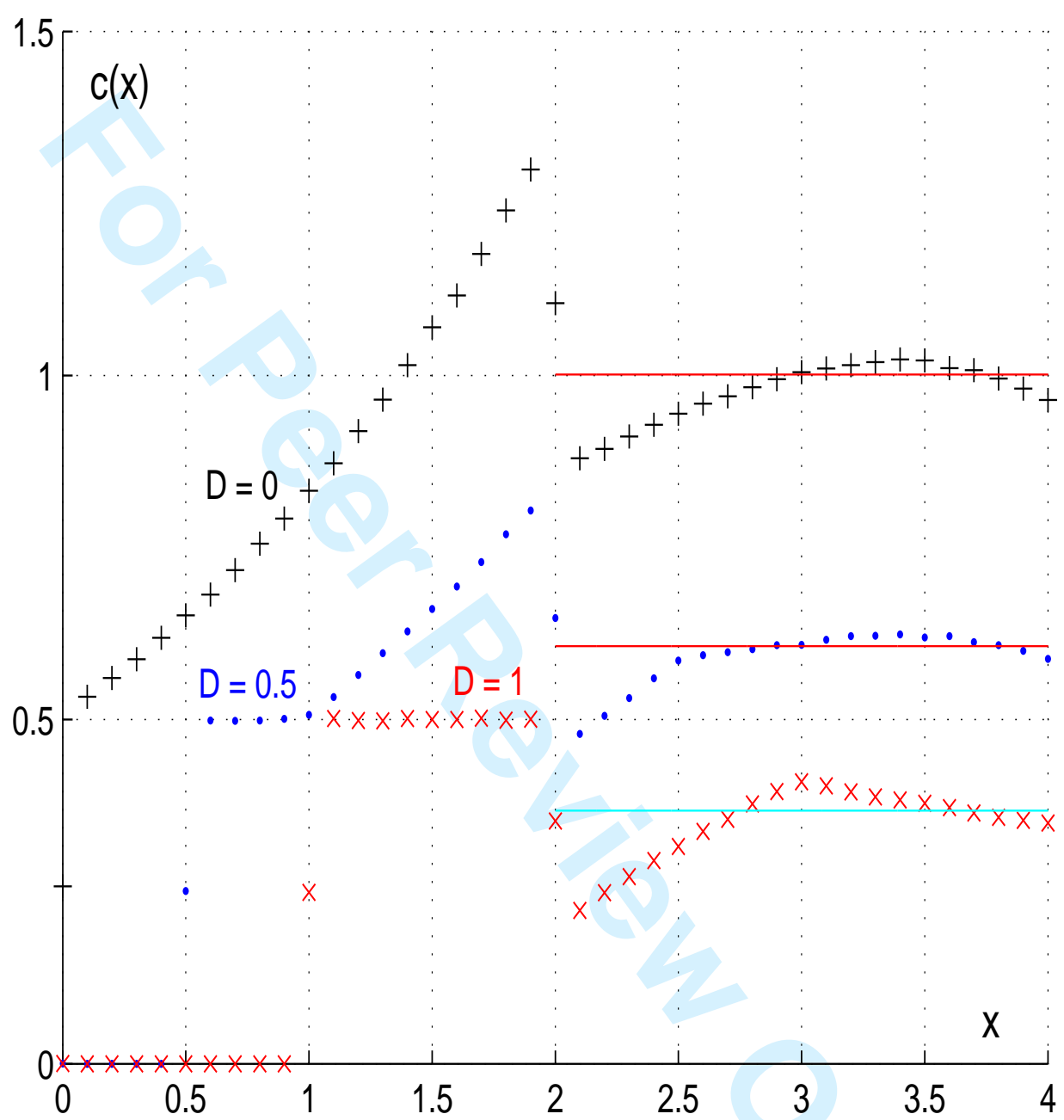

Figure 7: Coincidence function of a PP with uniform distribution and output DT for two values of $D$. 


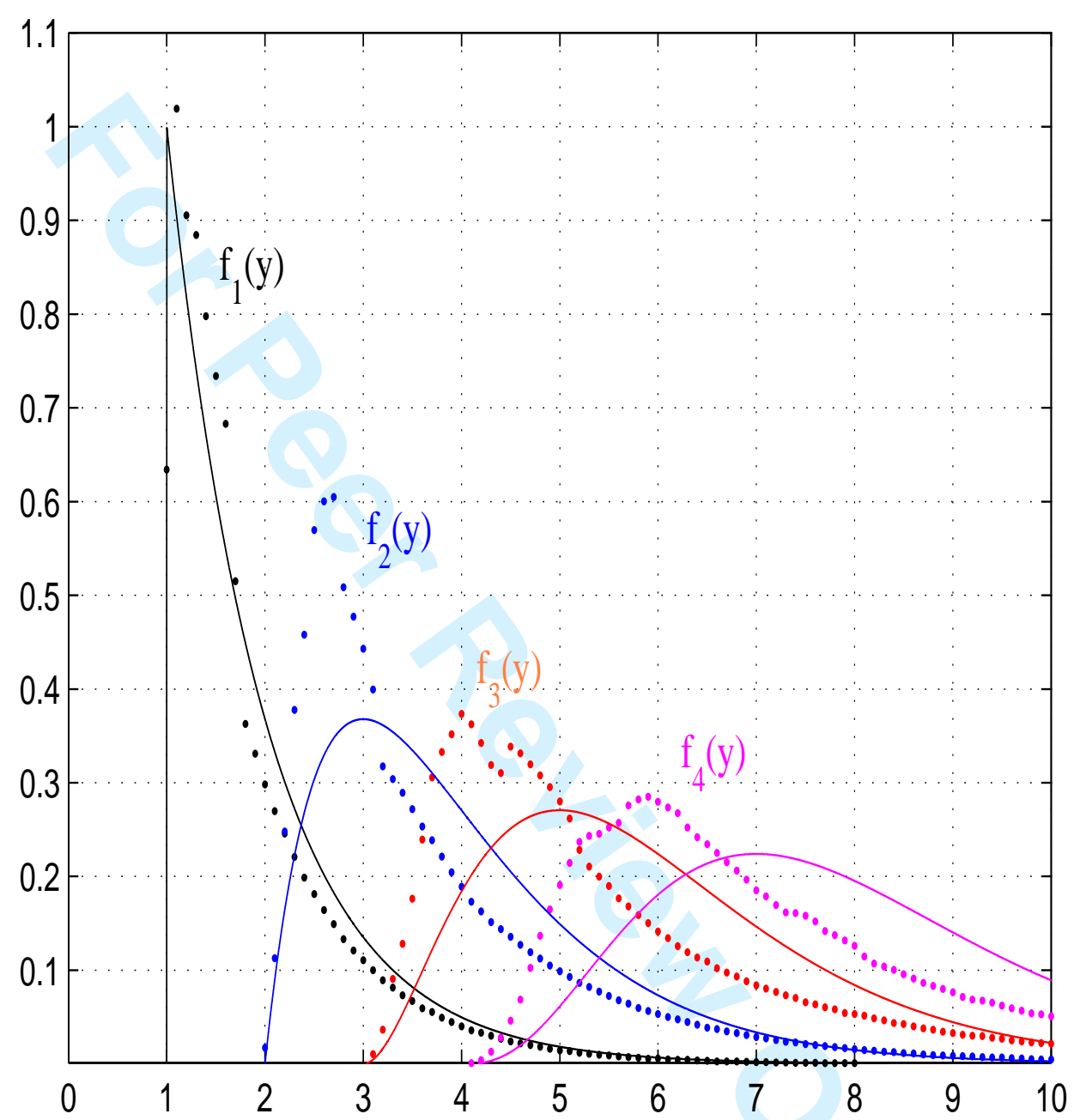

Figure 8: $\operatorname{PDFs} f_{1}(y), f_{2}(y), f_{3}(y), f_{4}(y)$ of a $\operatorname{EAR}(1) \mathrm{PP}$ with output DT, $D=1$. 


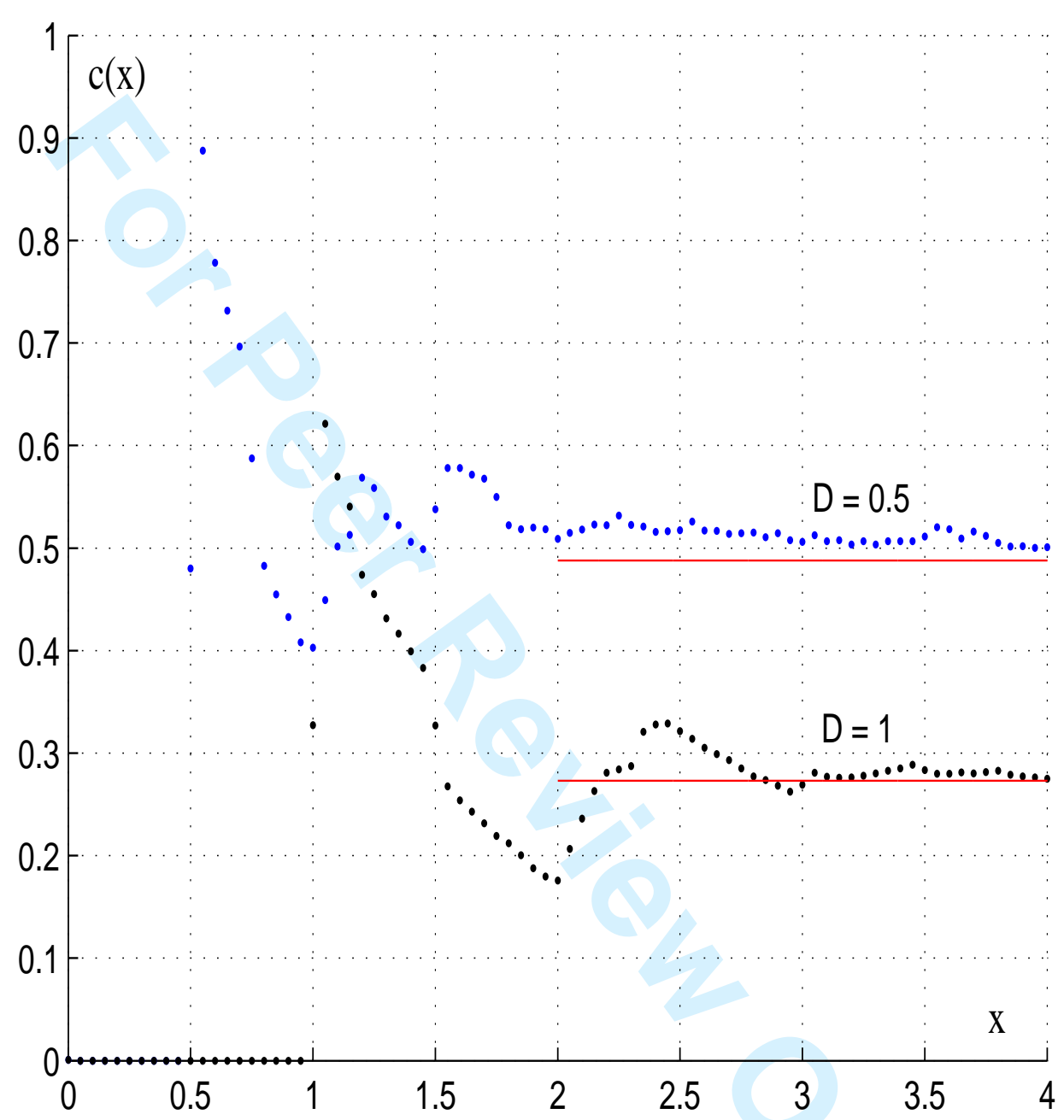

Figure 9: Coincidence function of a $\operatorname{EAR}(1) \mathrm{PP}$ with output DT for two values of D. $a=0.5$ 